\title{
WORK IN PROGRESS: Design Fixation in First-Year Engineering Students' Problem Solving
}

\section{DeLean Tolbert, Purdue University - West Lafayette}

DeLean Tolbert is an Engineering Education doctoral candidate at Purdue University. She earned a B.Sc. in Electrical Engineering from the University of Michigan-Dearborn and a M.S. in Industrial Engineering from the University of Michigan. Through her dissertation, DeLean investigates the ways that Black boys develop Engineer of 2020 attributes in their precollege out-of-school time lived experiences. This work will serve as a foundation for her future research, through which she anticipates exploring how ethnically diverse students apply these attributes to engineering tasks in both formal and informal settings.

\section{Dr. Monica E Cardella, Purdue University - West Lafayette}

Monica E. Cardella is the Director of the INSPIRE Research Institute for Pre-College Engineering Education and is an Associate Professor of Engineering Education at Purdue University. 


\title{
WORK IN PROGRESS: Design Fixation in First-Year Engineering Students' Problem Solving
}

\begin{abstract}
Idea generation is an essential phase of the engineering design process. It is during this phase that the engineer can develop many possible solutions to address the design constraints, which will later be reduced to the final design solution. However, in some cases engineers may experience design fixation, which could lead to limited creativity and limited design solutions.

In this study, design fixation is being explored as first-year engineering students work independently for three hours to design a playground for a fictitious neighborhood. The research team explored the initial idea generation phase of first-year engineering students $(\mathrm{N}=14)$ with differing mathematics and design precollege experiences. The results of the quantitative analysis suggest that first-year engineering students in this study can be considered a homogenous group with respect to their mean number of design solutions. The participant's previous mathematical and design knowledge and experiences did not moderate idea generation.
\end{abstract}

\section{Introduction}

Engineering and Design educators observe that at differing points students struggle with fixation. This is a concept which represents the premature commitment to an idea, solution or concept and can take many forms across disciplines. ${ }^{[1}$. Considering that engineering students typically solve problems which vary greatly in complexity, can fall anywhere on the continuum for definedness, from well-defined problems to ill-defined problems and which require them to access and apply knowledge from varying disciplines, the occurrence of design fixation in engineering students must be understood. Beyond understanding, engineering education and design researchers should investigate the occurrence of design fixation along with the ways students overcome fixation.

Some may argue that fixation is not necessarily the enemy of innovation or to reaching the ideal solution for the tasks. However, it is our belief that for some students, it can serve an inhibitor to reaching a final design within a given time constraint. This is important as most tasks are constrained if not by time then by other factors. Therefore, educators and students identify when fixation occurs and must overcome it. For example, the results from a study of engineers and 
fixation ${ }^{[1]}$ suggest two approaches to overcome fixation: 1) students should develop domain knowledge around a problem before solving to prevent from deferring to everyday knowledge and limit innovative solutions and 2) students should not focus solely on generating higher quantities of ideas as they risk fixation on innovation and developing many solutions which lack depth in disciplinary principles.

\section{Objectives}

This research team is investigating the ways students, with diverse levels of experience and knowledge in engineering, mathematics and design, solve problems. How do college students approach ambiguous tasks? Is there evidence of design fixation as they are solving engineering problems? Are there instances of convergent and divergent thinking in their design processes? What counts as evidence for these types of thinking? For inclusion in this publication, playground design task data were analyzed to investigate fixation by exploring the relationship between the number of ideas generated and their respective mathematics and design previous experiences for first-year engineering students (FYE).

Each participant works for three hours as they complete a design of a playground for a fictional neighborhood (see fig. 1). During the design sessions, the research team observed students accessing different sources of information: their own knowledge and experiences, the internet, and information from requested by the facilitator. The students draw from their own experiences as they reflect and record the pieces of equipment which they might remember playing on as youth. Students often say, "What did I play on when I was younger.... Oh that was fun. Yes, they (the fictional neighborhood children) would like that." With respect to the internet, many students search "playground equipment" and look at previous designs. They may also use the internet as a resource after brainstorming to ensure they have considered every possible piece of playground equipment. Finally, few students request information related to community wants and needs, beyond what is provided in the design task.

This paper will compare 14 students' idea generation- approaches with the purpose of investigating what similarities might exist between the students mathematical, design or engineering background and their design decisions. One of the hypotheses driving this study is that students with high-mathematics backgrounds would experience more design fixation than those students who are categorized as low-mathematics students. We will investigate this 
hypothesis by ability grouping the students and exploring their idea generation phase both individually and looking for differences and similarities across the groups.

For many of the students this sample, the idea generation phase took place within the first hour of the three-hour study. However, some students later developed more ideas later in the study after realizing constraints were not met. The later developed ideas are not the focus of this study.

\section{Conceptual Framework}

As of publication, this approved human subjects study is currently recruiting participants from engineering, design and mathematics majors. The objective is to investigate the guiding hypothesis by observing potential differences between groups of students with more or less design and mathematics experiences. The research team is building the investigation around a conceptual framework which include:

- Mathematical Modeling: a procedure (model) for solving the problem using multiple mathematical ideas. ${ }^{[2]}$

- Fixation: a premature commitment to a particular problem solution, blind adherence to one solution or one approach to a problem (mechanized thought), which follows a previously laid-out pattern, prevents the consideration of all relevant knowledge and experience which should be brought to bear on the given problem. ${ }^{[1,3]}$

- Divergent Thinking: imagining any possibility, heading off in many directions, deliberately diverge from the conventional, exploring possibilities while temporarily suspending criticism and judgment. ${ }^{[4]}$

- Convergent thinking: narrowing down the options to one or more choices. ${ }^{[4]}$

\section{Methods}

\section{Participants}

Fourteen first-year engineering students in a large mid-western university have participated in this study, as of publication. The students participated at different points during their first semester of college and differed in their mathematics and design experiences. Students were selected to represent diversity in sex, ethnicity and academic experiences. See Table 1 for demographic information. 
Table 1. The First Year Engineering Participants in the Research Study (N=14)

\begin{tabular}{|c|c|c|c|c|}
\hline $\begin{array}{c}\text { Participant } \\
\text { Pseudonym }\end{array}$ & Sex & Ethnicity & $\begin{array}{c}\text { Mathematics } \\
\text { Experience }\end{array}$ & $\begin{array}{c}\text { Design } \\
\text { Experience }\end{array}$ \\
\hline Kasira & F & White & High & $\checkmark$ \\
\hline Ann & F & White & High & $\checkmark$ \\
\hline Mark & M & White & High & $\checkmark$ \\
\hline April & F & White & High & $\checkmark$ \\
\hline Catharine & F & White & Low & $\checkmark$ \\
\hline Claudia & F & White & High & \\
\hline Julia & F & Hispanic & High & $\checkmark$ \\
\hline Jane & F & White & Low & \\
\hline Sarah & F & White & Low & \\
\hline Andrew & M & White & Low & \\
\hline Peter & M & White & Low & $\checkmark$ \\
\hline Ashley & F & White & Low & $\checkmark$ \\
\hline Tabitha & F & White & Low & \\
\hline Sabrina & F & Asian- American & Low & \\
\hline
\end{tabular}

\section{Design and Data Sources}

Each participant was allowed three hours to complete the design task. They were given the design task statement, a resource box (with miscellaneous items such as writing utensils, post-it notes, colored pencils, paper and rulers), and could request information cards from the facilitator. During this study, the students were encouraged to "think aloud" which aligns with the verbal protocol method. ${ }^{[6]}$ Each session was video and audio recorded, and field notes were taken by the facilitator at five minute intervals. The students' final design and all artifacts were also collected for later analysis.

In the pilot studies, students commented that the study experiences would more resemble real an actual engineering tasks if it were a group task. The research team addressed this concern by modifying previous methods of facilitating this playground design task to include the use of an internet accessible laptop computer in addition to the information cards. ${ }^{[5]}$

\section{Analysis}

The analysis of the data will occur in several phases. The objective of the first phase of analysis, which is the focus of this publication, is to test for the homogeneity of the numbers of ideas generated for playground equipment by the sample of first-year engineering students. The results 
of the statistical analysis will reveal if significant differences exist in the number of ideas generated by first-year engineering students with different mathematical and design backgrounds. Once it is determined that the group is homogeneous with respect to idea generation, all first-year engineering students will be grouped together and compared against other groups in the study. In order to assess fixation, a measurement of similarity approach will be used to evaluate the participants' final design solution (see fig. 2). In this work, the direct measure of fixation will be used to report the quantity of equipment ideas generated by each student. In future work, fixation will be explored by categorizing ideas as reproductive or analogical.

Table 2. Description of Measures of Similarity

\begin{tabular}{|l|l|}
\hline Direct & For this proposal, the results from the ANOVA analysis will be reported. \\
\hline Reproductive & Participants used parts of the example or configurations of parts that could be recognized \\
\hline Analogical & $\begin{array}{l}\text { Participants used design principles in the example designs without using the actual physical form of } \\
\text { the example }\end{array}$ \\
\hline
\end{tabular}

During each student's idea generation phase, each instance of a new idea was recorded. Students' verbalized rationale for the idea was also used to understand the idea generation process. For analysis purposes, the participants were ability grouped based on their mathematical and design experiences. According to the FYE advising office at the university under study, most incoming students have taken a Calculus I course in high school and are enrolled in Calculus II or its equivalent. Those students were placed in the "high mathematics group." Students with below normal mathematics backgrounds were enrolled in Calculus I as of their participation in this study and were grouped as "low mathematics."

The data were analyzed using a single factor ANOVA with the participants' ability (mathematics or design) as the independent measure. This method was selected in order to determine if the overall number of ideas generated was different between students with differing mathematical and design backgrounds. Mathematics levels are characterized as high and low. For this phase of analysis all of the participants are engineering students and therefore have very similar academic profiles as it relates to mathematics. This paper is a work in progress and only the first-year engineering students are included in the analysis and discussion. Therefore, this phase represents high performers. Future analyses will include data from senior engineering, design, and mathematics students, and additional first-year engineering students. The diversity of participants disciplines groups justifies the high and low mathematics distinctions for future analysis. 
Progress on this work, including the analysis of other student samples (i.e. design, senior engineering, and mathematic majors), will be discussed in future publications and presentations.

\section{Results}

The research team first determined the number of ideas generated by each of the 14 participants in the sample and then ability grouped the students. The one factor ANOVA was done with a significance level of 0.05 . The mean number of ideas generated for the high and low mathematics groups and the experience or no experience design groups were $9.00(S D=3.46)$, $8.13(S D=4.79), 7.88(S D=3.09)$, and $9.33(S D=5.47)$, respectively. The ANOVA revealed no differences between the mean number of ideas generated by the high and low mathematics groups and the design experience or no design experience groups. The results for the math ability grouping are as follows: $F_{(1,12)}=0.14, p=.71$. The results for the design experiences are: $F_{(1,}$ 12) $=0.49, p=.54$. Thus given the results, there is no evidence that there are differences in the means across the groups.

\section{Significance}

The primary purpose of this research investigation was to explore idea fixation by first examining the idea generation phase of 14 first-year engineering students. This was accomplished by analyzing the data 1) to determine the number of ideas generated by $14 \mathrm{FYE}$ students and 2) to explore differences and similarities between groups of FYE students with diverse mathematical and design backgrounds. The ANOVA approach showed no statistically significant difference in the means of participants' idea generation with respect to the mathematics and design ability groups. For the purposes of our investigation, all first-year engineering students, regardless of previous exposure can be considered homogenous and can be grouped together for comparison against other groups with more or less mathematics and design experiences. As more data is collected, the research team will further this investigation in order to prove or disprove our guiding hypothesis that a student's mathematics background moderates design fixation in engineering design tasks.

\section{Future work}

Data collection for first-year engineering students has been completed. The research team is currently, refining the coding scheme which will be used to analyze the video recordings of the study session and can help identify design fixation. As we build upon our understanding of the 
role of fixation in engineering students' design process, measures of similarity (direct, reproductive, and analogical) can be used to identify instances of fixation in each participant's final design. ${ }^{[7]}$ Fixation can then be analyzed by looking for measures of similarity in each participant's final design solution. Using a similar analysis approach as Chrysikou ${ }^{[7]}$, the time to complete each of the required solutions will be recorded. The video and audio data will be used to clarify uncertainties in the final design solution. We can use the approach discussed in this paper along with the process for determining measures of fixation to make comparisons between first-year engineering students and other groups (i.e. engineering, design and mathematics seniors).

\section{Acknowledgement}

This material is based upon work supported by the National Science Foundation under Grant No. EEC-1151019. Any opinions, findings, and conclusions or recommendations expressed in this material are those of the authors and do not necessarily reflect the views of the National Science Foundation. We would also like to acknowledge the contributions of Kelly-Ann Dixon Hamil, a Purdue Statistics graduate student and Dr. Monique Long, University of Texas - San Antonio research associate.

\section{Reference}

[1] Purcell, A.T., and J.S. Gero," Design and other types of fixation", Design Studies Vol. 17, No. 4, 2006, pp. 363-383.

[2] Diefes-Dux, H.A., J.S. Zawojewski, M.A. Hjalmarson, and M.E. Cardella," A framework for analyzing feedback in a formative assessment system for mathematical modeling problems", Journal of Engineering Education Vol. 101, No. 2, 2012, pp. 375-406.

[3] Jansson, D.G., and S.M. Smith," Design Fixation", Design Studies Vol. 12, No. 1, 1991, pp. 311.

[4] Dym C. L., Agogino A. M., Eris O., D.D. Frey, and L. Leifer," Engineering design thinking, teaching, and learning", Journal of Engineering Education Vol. 94, No. 1, 2005, pp. 103-120.

[5] Atman, C.J., R.S. Adams, M.E. Cardella, J. Turns, S. Mosborg, and J. Saleem," Engineering Design Processes: A Comparison of Students and Expert Practitioners", Journal of Engineering Education Vol. 96, No. 4, 2007, pp. 359-379.

[6] Atman, C.J., and K.M. Bursic," Verbal protocol analysis as a method to document engineering student design processes", Journal of Engineering Education Vol. 87, No. 2, 1998, pp. 121-132.

[7] Chrysikou, E.G., and R.W. Weisberg," Following the Wrong Footsteps: Fixation Effects of Pictorial Examples in a Design Problem-Solving Task", Journal of Experimental Psychology: Learning, Memory, and Cognition Vol. 31, No. 5, 2005, pp. 1134-1148. 